\title{
Screening SIRT1 Activators from Medicinal Plants as Bioactive Compounds against Oxidative Damage in Mitochondrial Function
}

\author{
Yi Wang, ${ }^{1}$ Xinying Liang, ${ }^{1}$ Yaqi Chen, ${ }^{1}$ and Xiaoping Zhao ${ }^{2}$ \\ ${ }^{1}$ College of Pharmaceutical Sciences, Zhejiang University, Hangzhou 310058, China \\ ${ }^{2}$ College of Preclinical Medicine, Zhejiang Chinese Medical University, Hangzhou 310053, China
}

Correspondence should be addressed to Yi Wang; mysky@zju.edu.cn and Xiaoping Zhao; zhaoxiaoping@zcmu.edu.cn

Received 20 October 2015; Revised 29 December 2015; Accepted 12 January 2016

Academic Editor: Hesham A. El Enshasy

Copyright (c) 2016 Yi Wang et al. This is an open access article distributed under the Creative Commons Attribution License, which permits unrestricted use, distribution, and reproduction in any medium, provided the original work is properly cited.

\begin{abstract}
Sirtuin type 1 (SIRT1) belongs to the family of $\mathrm{NAD}^{+}$dependent histone deacetylases and plays a critical role in cellular metabolism and response to oxidative stress. Traditional Chinese medicines (TCMs), as an important part of natural products, have been reported to exert protective effect against oxidative stress in mitochondria. In this study, we screened SIRT1 activators from TCMs and investigated their activities against mitochondrial damage. 19 activators were found in total by in vitro SIRT1 activity assay. Among those active compounds, four compounds, ginsenoside $\mathrm{Rb}_{2}$, ginsenoside $\mathrm{F} 1$, ginsenoside $\mathrm{Rc}$, and schisandrin $\mathrm{A}$, were further studied to validate the SIRT1-activation effects by liquid chromatography-mass spectrometry and confirm their activities against oxidative damage in H9c2 cardiomyocytes exposed to tert-butyl hydroperoxide (t-BHP). The results showed that those compounds enhanced the deacetylated activity of SIRT1, increased ATP content, and inhibited intracellular ROS formation as well as regulating the activity of Mn-SOD. These SIRT1 activators also showed moderate protective effects on mitochondrial function in t-BHP cells by recovering oxygen consumption and increasing mitochondrial DNA content. Our results suggested that those compounds from TCMs attenuated oxidative stress-induced mitochondrial damage in cardiomyocytes through activation of SIRT1.
\end{abstract}

\section{Introduction}

Sirtuin type 1 (SIRT1) belongs to the family of class III histone deacetylases (HDAC) that consume $\mathrm{NAD}^{+}$during deacylation cycle. It has been reported that, in mammals, SIRT1 plays a critical function in cellular metabolism and response to oxidative stress [1-4].

Recently, researchers have found that SIRT1 activators can protect mitochondrial function from oxidative-induced mitochondrial damage in various types of cell through regulating PGC- $1 \alpha$ and multiple transcription factors [59], which are tightly related to mitochondrial biogenesis and metastasis $[10,11]$. It is also reported that activators of SIRT1, such as resveratrol [12], can extend lifespan and regulate metabolic disorders [13-15]. Therefore, SIRT1 activators exhibit unique pharmacological potentials for treating mitochondrial dysfunction related diseases. Meanwhile, several clinical trials of SIRT1 activators such as SRT1720 for type 2 diabetes and obesity are under way [16].

Natural products have historically been regarded as an important resource of therapeutic agents in pharmaceutical discovery over the past century [17]. Traditional Chinese medicines (TCMs), as an important part of natural products, are mainly governed by empirical experience and fundamental theories such as the Yin and Yang concept [18]. TCMs with Qi Tonification effects including Astragalus membranaceus $[19,20]$, Panax ginseng $[21,22]$, and Panax notoginseng [23, 24] have been reported to exert protective effect against oxidative stress in mitochondria. Several compounds isolated from TCMs are reported to regulate SIRT1 activity [25]. However, a comprehensive screening of SIRT1 activators from TCMs has not yet been performed to investigate their protective effects on mitochondrial function against oxidative stress. 
The aim of present study is to discover SIRT1 activators from TCMs and validate their activities against mitochondrial damage. A sensitive in vitro assay to screen SIRT1 activators was performed to discover bioactive compounds from TCMs, and the lead compounds were validated by liquid chromatography-mass spectrometry (LC-MS) analysis. Effects of identified SIRT1 activators on mitochondrial function were further investigated in cardiomyocytes exposed to tert-butyl hydroperoxide (t-BHP). ATP content, intracellular ROS formation, and activity of Mn-SOD were measured. Moreover, oxygen consumption and mitochondrial DNA content of cardiomyocytes were used to evaluate the effects of those SIRT1 activators on mitochondrial function.

\section{Materials and Methods}

2.1. Supplies and Chemicals. SIRT1 protein (human recombinant) and lysyl endopeptidase were purchased from Cayman Chemical (USA). Ginsenoside F1, ginsenoside Rc, and schisandrin A were purchased from Shanghai Winherb Medical Technology Company (China). Ginsenoside $\mathrm{Rb}_{2}$ was purchased from National Institute for Food and Drug control (Beijing, China).

2.2. Cell Culture. H9c2 (from Cell Bank of Chinese Science Academy, Shanghai, China) were cultured in DMEM (Corning, USA) containing 10\% fetal bovine serum (Sigma, USA), $100 \mathrm{U} / \mathrm{mL}$ penicillin, and $100 \mu \mathrm{g} / \mathrm{mL}$ streptomycin (Gibco, USA). All the cells were grown in 5\% humidified $\mathrm{CO}_{2}$ atmosphere at $37^{\circ} \mathrm{C}$.

2.3. Fluorescent Probe Based Assay for SIRT1 Modulation Effects of Compounds. The measurement of SIRT1 activity and effects of compounds on SIRT1 activation were performed by a previously reported fluorescent method [26]. Briefly, SIRT1 was incubated with a TPE-GK(Ac)YDD probe $(20 \mu \mathrm{M})$ in the presence of the tested compound $(50 \mu \mathrm{M})$. Fluorescence intensity was recorded by a TECAN infinite F200 microplate reader with excitation wavelength $320 \mathrm{~nm}$ and emission wavelength $465 \mathrm{~nm}$.

A total of 195 constituents of TCMs were screened by the assay to evaluate their regulatory effects on SIRT1 activity. The detailed information related to the chemicals and their CAS number were illustrated in Supplementary Material available online at http://dx.doi.org/10.1155/2016/4206392. The inhibition or activation of SIRT1 was calculated by the following equation:

$$
\operatorname{Activation} \operatorname{rate}(\%)=\left(\frac{\left(I_{s}-I_{s 0}\right) / I_{s 0}}{\left(I_{c}-I_{0}\right) / I_{0}}-1\right) \text {. }
$$

$I_{s}$ and $I_{c}$ represented the fluorescence intensity of tested sample group with various test compounds and control group without test compounds. $I_{s 0}$ and $I_{0}$ represented the fluorescence intensity of tested sample and control when incubated without SIRT1 protein. To explore the dose-related effects of these compounds, several active compounds, including ginsenoside $\mathrm{Rb}_{2}$, ginsenoside $\mathrm{Rc}$, and schisandrin $\mathrm{A}$, were further tested with the concentrations of $1,10,25$, and $50 \mu \mathrm{M}$.
2.4. Validation of SIRT1 Activators by LC-MS Analysis. In order to validate activation of SIRT1, SIRT1 $(10 \mu \mathrm{g} / \mathrm{mL})$ was added to TPE-GK(Ac)YDD $(20 \mu \mathrm{M})$ and $\mathrm{NAD}^{+}$(Sigma, $3 \mathrm{mM}$ ) for $1 \mathrm{~h}$ incubation at $37^{\circ} \mathrm{C}$ in the presence or absence of ginsenoside $\mathrm{Rb}_{2}$, ginsenoside $\mathrm{F} 1$, ginsenoside $\mathrm{Rc}$, and schisandrin A $(50 \mu \mathrm{M})$, respectively. Samples were boiled at $100^{\circ} \mathrm{C}$ for $10 \mathrm{~min}$ to degenerate SIRT1 and terminate reaction.

Samples were analyzed by Agilent 1100 LC system (Agilent Technologies, USA) and Finnigan LCQ Deca XP ${ }^{\text {plus }}$ ion trap mass spectrometer with an ESI source (Thermo, USA). The acquisition parameters for LC/ESI-MS were as follows: nebulizing gas, high-purity nitrogen $\left(\mathrm{N}_{2}\right)$; collision gas, high-purity helium (He); capillary voltage: $-15 \mathrm{~V}$; capillary temperature: $350^{\circ} \mathrm{C}$; ion spray voltage: $-3 \mathrm{kV}$; tube lens offset voltage: $-30 \mathrm{~V}$; mass range: $\mathrm{m} / z$ 100-1500. Chromatographic separation was performed by a reversed-phase ZORBAX SB$\mathrm{C}_{18}$ analytical column. The mobile phase included water containing $0.1 \%(\mathrm{v} / \mathrm{v})$ formic acid (A) and acetonitrile (B). The flow rate was $0.6 \mathrm{~mL} / \mathrm{min}$. A gradient program was carried out as the following profile: $0 \mathrm{~min}, 50 \% \mathrm{~B} ; 5 \mathrm{~min}, 50 \%$ B; $30 \mathrm{~min}, 95 \% \mathrm{~B} ; 40 \mathrm{~min}, 95 \% \mathrm{~B}$.

2.5. Measurement of ATP Content and Intracellular ROS. H9c2 cells were seeded in 96 wells at the density of 4,000/well. The cells were preincubated with ginsenoside $\mathrm{Rb}_{2}$, ginsenoside F1, ginsenoside Rc, and schisandrin A $(20 \mu \mathrm{M})$ for $24 \mathrm{~h}$ before being exposed to t-BHP $(300 \mu \mathrm{M})$ for $1 \mathrm{~h}$. Intracellular ATP content was measured by CellTiter-Glo ${ }^{\circledR}$ Luminescent Assay kit (Promega) according to the instruction of manufacture. Intracellular ROS content was measured by DCFH-DA probe $(5 \mu \mathrm{M})$ whilst fluorescence intensity was recorded by a TECAN infinite F200 Multifunction microplate with excitation wavelength $485 \mathrm{~nm}$ and emission wavelength $535 \mathrm{~nm}$. The changes of ATP content and ROS accumulation were calculated by comparing the luminescent or fluorescent signal of the treated cells with that of untreated H9c2 cells.

2.6. Detection of Mn-SOD Activity. Manganese superoxide dismutase (Mn-SOD) was an antioxidative enzyme, which protected against ROS-induced damage [27]. To measure Mn-SOD activity, $\mathrm{H} 9 \mathrm{c} 2$ cells were seeded in 6-well plate in the density of $4 \times 10^{5} / \mathrm{mL}$. H9c2 cells were preprotected for $24 \mathrm{~h}$ by ginsenoside $\mathrm{Rb}_{2}$, ginsenoside $\mathrm{F} 1$, ginsenoside $\mathrm{Rc}$, and schisandrin A $(20 \mu \mathrm{M})$ before being exposed to t-BHP $(300 \mu \mathrm{M})$ for $1 \mathrm{~h}$. The cells were lysed and the concentration of total protein was measured by BCA assay kit. Mn-SOD activity in total protein was measured by Mn-SOD assay kit (Beyotime, China).

2.7. Oxygen Consumption Assay. $2 \times 10^{6} \mathrm{H} 9 \mathrm{c} 2$ cells were seeded in $100 \mathrm{~mm}$ culture plate. After being grown to stable attachment, cells were preincubated with ginsenoside $R b_{2}$, ginsenoside $\mathrm{F} 1$, ginsenoside $\mathrm{Rc}$, and schisandrin $\mathrm{A}$ at final concentrations $(20 \mu \mathrm{M})$ for $18 \mathrm{~h}$ before being exposed to $\mathrm{t}$ BHP $(100 \mu \mathrm{M})$ for $1 \mathrm{~h}$. Cells were washed with PBS twice, subsequently collected by trypsinization followed by centrifugation, and resuspended in fresh medium. Respiratory activity was measured with a Clark-type oxygen electrode 
(Oxytherm, Hansatech Instruments, UK). An aliquot ( $1 \mathrm{~mL}$ ) of suspended cells $\left(2 \times 10^{6}\right.$ cells $\left./ \mathrm{mL}\right)$ was placed in the airtight liquid-phase oxygen electrode chamber. The system was maintained at $37^{\circ} \mathrm{C}$. After equilibration, the slope of oxygen consumption in $\mathrm{H} 9 \mathrm{c} 2$ cells was measured. Every $1 \times 10^{6}$ cells oxygen consumption was calculated as the basic respiration rate of each group. The experiment was also performed in the presence of SIRT1 inhibitor, EX527 at final concentration of $20 \mu \mathrm{M}$ to investigate whether the effect of ginsenoside $\mathrm{Rb}_{2}$ can be prevented by SIRT1 inhibitor.

2.8. Measurement of Mitochondrial DNA Content. Real-time PCR was used to determine relative quantities of mitochondrial DNA content in $\mathrm{H} 9 \mathrm{c} 2$ cells exposed to t-BHP and cells incubated with SIRT1 activators. Cells were preincubated for $24 \mathrm{~h}$ with ginsenoside $\mathrm{Rb}_{2}$, ginsenoside $\mathrm{Fl}$, ginsenoside $\mathrm{Rc}$, and schisandrin $\mathrm{A}$ at final concentrations $(20 \mu \mathrm{M})$ before being exposed to t-BHP $(300 \mu \mathrm{M})$ for $1 \mathrm{~h}$. Cells in normal condition were used as control group. Total DNA was extracted using Mammalian Genomic DNA Miniprep Kits (Sigma, USA). DNA was quantified by measuring $A_{260}$ values, and $50 \mathrm{ng}$ of total DNA was used for PCRs by GenElute $^{\mathrm{Tx}}$ QuantiFast SYBR Green PCR Kit (QIAGEN, Germany). Primers specific to the mitochondrial-encoded Atp6 gene (Fw: 5'-ATT ACG GCT CCT GCT CAT A-3'; Rev: $5^{\prime}$-TGG CTC AAC CAA CCT TCT A-3') were used to assess mitochondrial DNA copy numbers. Primers designed against the nuclear-encoded Rpl13 gene (Fw: $5^{\prime}$-CAC AAG AAA ATG GCA CGC AC-3'; Rev: $5^{\prime}$-GAG CAG AAG GCT TCC TGG G-3 ${ }^{\prime}$ ) were used for normalization. $C_{T}$ values were obtained automatically. The number of mitochondrial genes was calculated by $2^{-\Delta \Delta C_{T}}$ method.

2.9. Statistical Analysis. All values were expressed as the means $\pm \mathrm{SD}$. One way ANOVA was used to analyze differences among groups. Statistical analysis was performed using GraphPad Prism. $p$ values of less than 0.05 were considered statistically significant.

\section{Results}

3.1. Screening of SIRT1 Activators from Compound Library of TCM. To screen SIRT1 activators, we chose 195 compounds from TCMs with different efficacy, which were defined according to the TCMs theory. For example, Panax ginseng and Ophiopogon japonicas were regarded as Tonifying herbs, whilst Schisandra chinensis was Astringent herb and Sophora flavescens came from heat-clearing medicinal. Figure 1 exhibited a heatmap of activation or inhibition rate of each compound. The corresponding values were listed in Supplementary Table. A total of 19 SIRT1 activators were found, including 20(S)-ginsenoside $\mathrm{Rg}_{3}(60 \%)$, ginsenoside $\mathrm{Rb}_{3}$ (28\%), ginsenoside $\mathrm{F} 1$ (22\%), and ginsenoside F2 (45\%) from Panax ginseng, gypenoside XVII (43\%) and notoginsenoside Ft1 (40\%) from Panax notoginseng, polyphyllin I (24\%), polyphyllin III (32\%), polyphyllin VI (31\%), and polyphyllin VII (32\%) from Paris polyphylla, liriopesides B (65\%) and Liriope muscari baily saponins C (57\%) from
Liriope muscari, ophiopogonin $\mathrm{D}^{\prime}$ (54\%) from Ophiopogon japonicas, saikosaponin A (25\%) from Bupleurum chinense, schisandrin B (30\%) from Schisandra chinensis, and anisodine hydrobromide (60\%) from Anisodus tanguticus. It was obvious that most of identified activators belonged to Tonifying herb. Moreover, potential inhibitors of SIRT1 almost belonged to herbs with heat-clearing efficacy.

Representative compounds were chosen to validate their activation effects on SIRT1 activity. Ginsenoside $\mathrm{Rb}_{2}$ showed $8 \%$ to $152 \%$ activation with the concentration range of $1 \sim$ $50 \mu \mathrm{M}$. Ginsenoside Rc exerted $88 \%$ activation at the concentration of $50 \mu \mathrm{M}$. Schisandrin A showed $28 \%$ activation at the concentration of $50 \mu \mathrm{M}$.

3.2. Validation of SIRT1 Activators by LC-MS Analysis. To validate the SIRT1-activation effect of the compounds, liquid chromatography-mass spectrometry (LC-MS) analysis was employed. The specific substrate of SIRT1, TPE-GK(Ac)YDD (Figure 2(a)), was incubated with SIRT1 $(10 \mu \mathrm{g} / \mathrm{mL})$ and $\mathrm{NAD}^{+}(3 \mathrm{mM})$ for $1 \mathrm{~h}$. The reaction product was identified by LC-MS based on the molecular weight. As shown in Figure 2(b), the deacetylated peptide, TPE-GKYDD, was detected with a loss of Ac ( $43 \mathrm{Da})$. When analyzing the deacetylated reaction in the presence of SIRT1 activators, including ginsenoside $\mathrm{Rb}_{2}$, ginsenoside $\mathrm{Fl}$, ginsenoside $\mathrm{Rc}$, and schisandrin $\mathrm{A}$, the peaks of deacetylated product were significantly elevated (Figure 2(c)). Our findings indicated that these compounds activated SIRT1 in enzymatic reaction.

3.3. Effects of SIRT1 Activators on Cardiomyocytes Oxygen Consumption. The effects of SIRT1 activators on mitochondrial function were further investigated by measuring cellular respiration in $\mathrm{H} 9 \mathrm{c} 2$ cells. As shown in Figure 3(a), basal respiration of t-BHP treated cardiomyocytes was significantly dropped comparing with control group. Preincubation with ginsenoside $R b_{2}$, ginsenoside $F 1$, ginsenoside $R c$, and schisandrin $\mathrm{A}$ attenuated the decrease of oxygen consumption. Figure 3(b) showed the representative oxygen consumption slope of normal cells $\left(1.65 \pm 0.31 \mathrm{nmol} \mathrm{O}_{2} / \mathrm{mL} / \mathrm{min}\right)$, tBHP treated cells $\left(1.10 \pm 0.25 \mathrm{nmol} \mathrm{O}_{2} / \mathrm{mL} / \mathrm{min}\right)$, and ginsenoside $\mathrm{Rb}_{2}$ treated cells $\left(1.43 \pm 0.25 \mathrm{nmol} \mathrm{O}_{2} / \mathrm{mL} / \mathrm{min}\right)$. The results suggested that these SIRT1 activators recovered the oxygen consumption rate in t-BHP injured cardiomyocytes. In the presence of SIRT1 inhibitor EX527 [28, 29], the protective effect of ginsenoside $\mathrm{Rb}_{2}$ was blocked, which indicated that the effect of ginsenoside $\mathrm{Rb}_{2}$ to recover the oxygen consumption rate was SIRT1 dependence (Figure 3(c)).

\subsection{Effects of SIRT1 Activators on ATP Content and ROS Accu-} mulation. As a specific parameter of mitochondrial function, intracellular ATP contents in cardiomyocytes exposed to oxidative stress were measured. After cells were exposed to $\mathrm{t}$-BHP for $1 \mathrm{~h}$, the content of ATP was significantly decreased. As shown in Figure 4, preprotection of cells by ginsenoside $\mathrm{Rb}_{2}$, ginsenoside $\mathrm{Fl}$, ginsenoside $\mathrm{Rc}$, and schisandrin A led to the recovery of ATP content, suggesting that those SIRT1 activators reversed the decreased mitochondrial energy metabolism induced by t-BHP. 


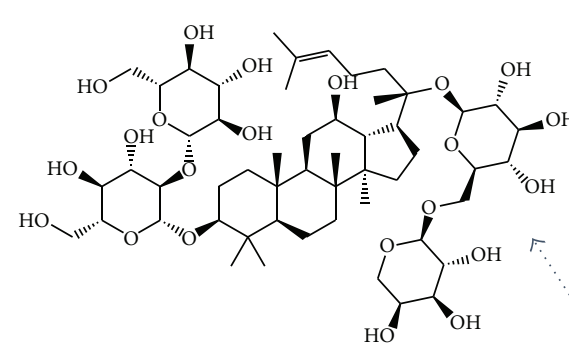
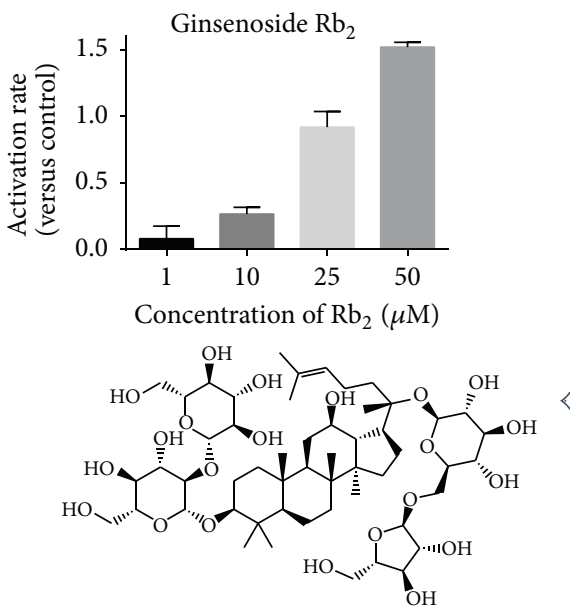

Ginsenoside Rc

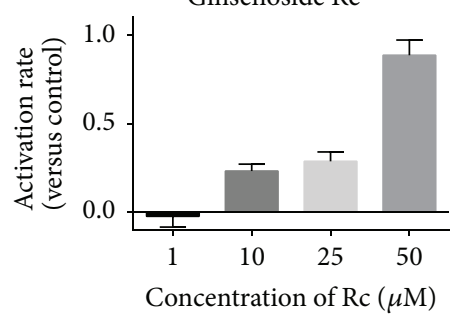

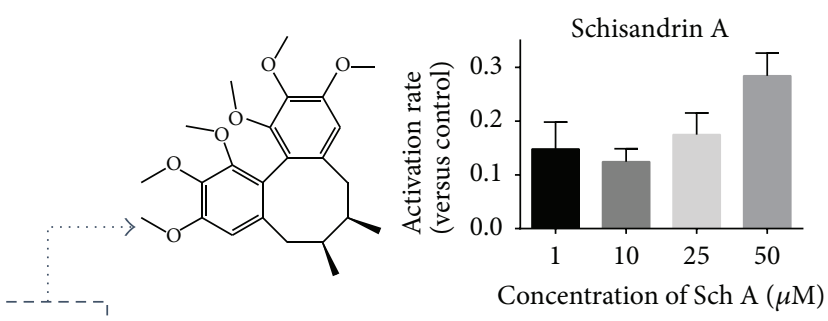

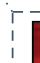

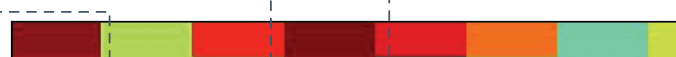

Concentration of Sch A $(\mu \mathrm{M})$

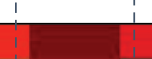

(1)
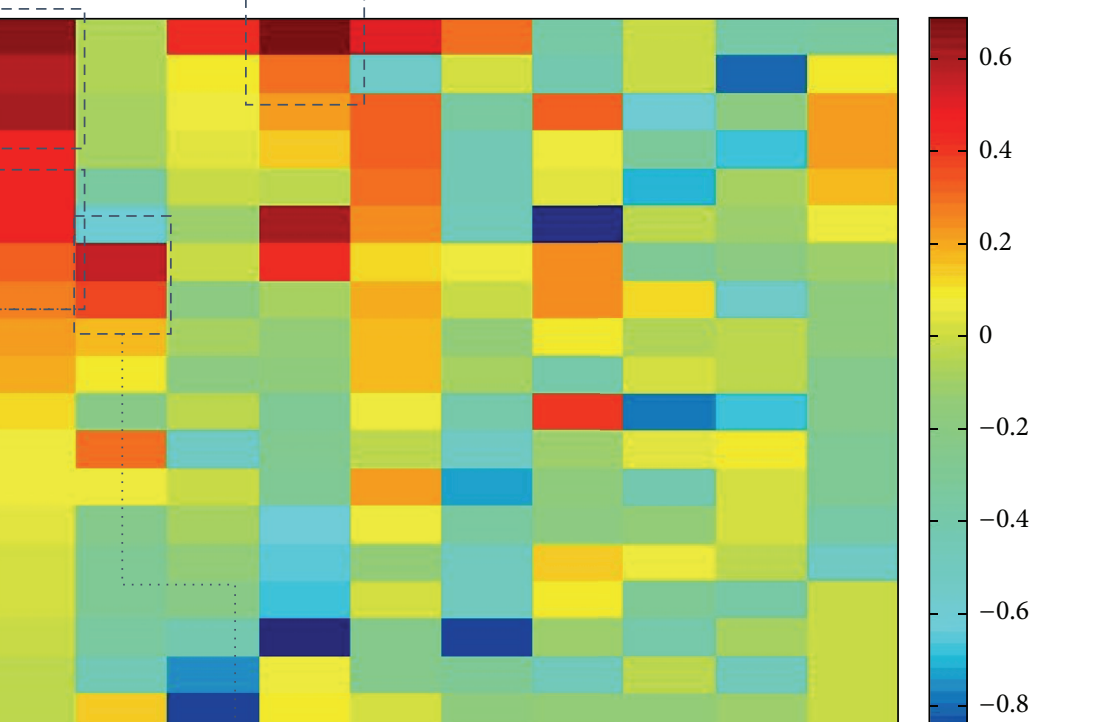

$-0.4$

0.2
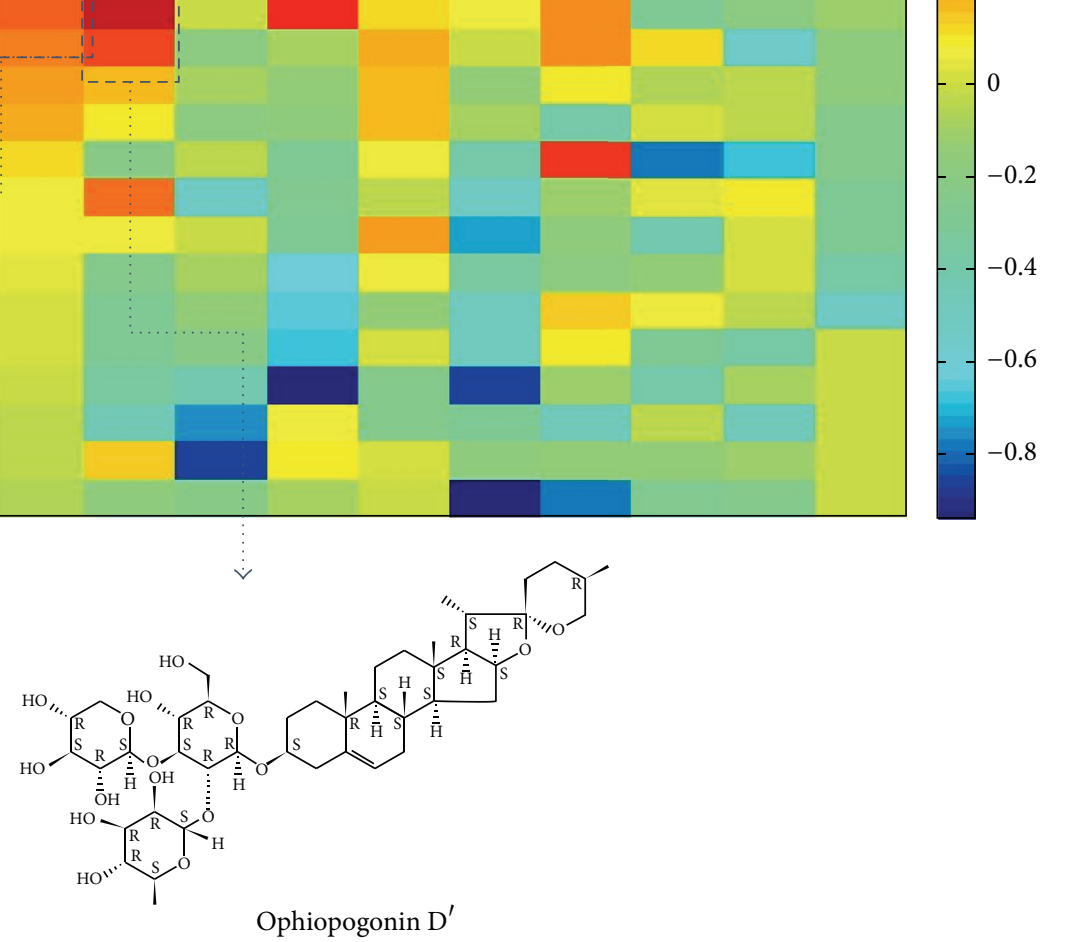

Ophiopogonin $\mathrm{D}^{\prime}$

FIGURE 1: Screening results of 195 compounds from TCMs and dose dependent activation of representative activators.

Mitochondrial oxidative stress was often caused by increased intracellular ROS formation. Figure 5 showed that the intracellular ROS was significantly increased after $t$ BHP treatment. Pretreatments of SIRT1 activators, ginsenoside $\mathrm{Rb}_{2}$, ginsenoside $\mathrm{Fl}$, ginsenoside $\mathrm{Rc}$, and schisandrin A, kept intracellular ROS levels on the normal condition.

3.5. Effects of SIRT1 Activators on Mn-SOD Activity. Mn-SOD was one of the antioxidative enzymes in mitochondria that assured mitochondrial oxidative stress resistance. As shown in Figure 6, when cells were exposed to t-BHP $(300 \mu \mathrm{M})$ for $1 \mathrm{~h}$, activity of Mn-SOD was decreased. Preprotection of cells by ginsenoside $R b_{2}$, ginsenoside $F 1$, ginsenoside $R c$, and schisandrin A enhanced the activity of Mn-SOD compared with t-BHP group.
3.6. Effects of SIRT1 Activators on Mitochondrial DNA Content. To verify the effect of ginsenoside $\mathrm{Rb}_{2}$, ginsenoside $\mathrm{F} 1$, ginsenoside Rc, and schisandrin A of mitochondrial protection or biogenesis, cells were injured by t-BHP after compounds preprotection, and then mitochondrial DNA content was analyzed. As shown in Figure 7, t-BHP treatment reduced mitochondrial DNA content compared to control group. Pretreatments of ginsenoside $\mathrm{Rb}_{2}$, ginsenoside $\mathrm{F} 1$, ginsenoside Rc, or schisandrin A significantly elevated the mitochondrial DNA content. Our findings suggested that these natural SIRT1 activators facilitated mitochondrial biogenesis.

\section{Discussion}

In the present study, we screened and identified 19 SIRT1 activators, such as ginsenoside $\mathrm{Rg}_{3}$, ginsenoside $\mathrm{Rb}_{2}$, ginsenoside 


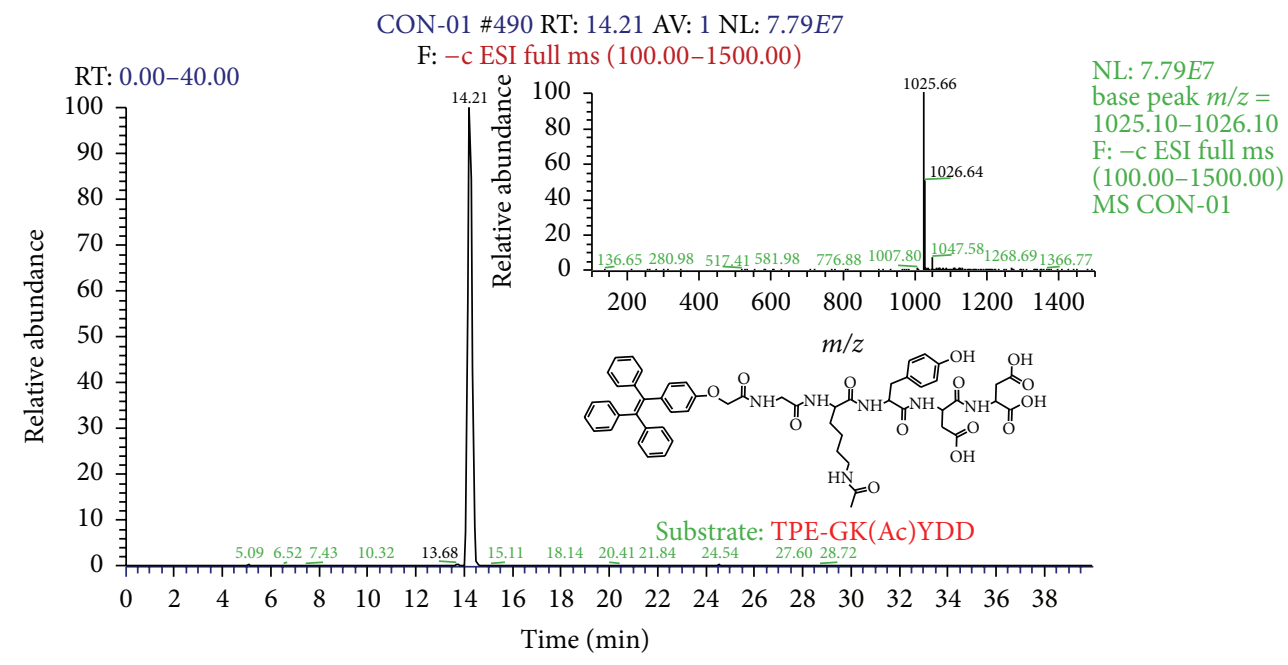

(a)

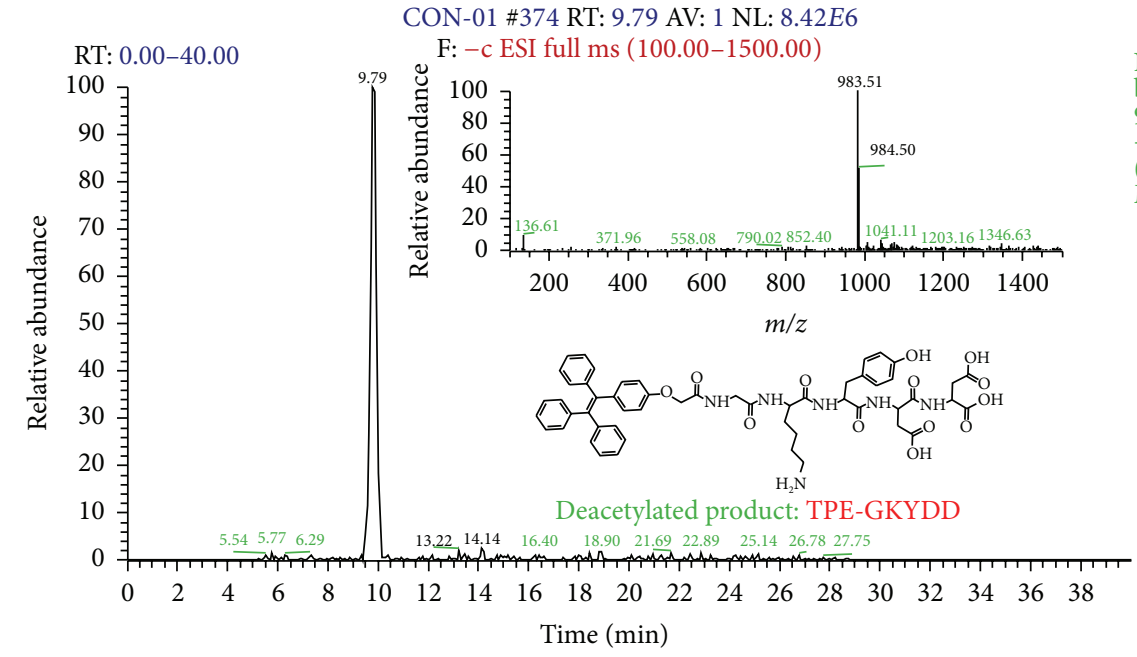

NL: $8.42 E 6$

base peak $m / z=$ 982.90-983.90 F:

$-\mathrm{c}$ ESI full ms

$(100.00-1500.00)$

MS CON-01

(b)

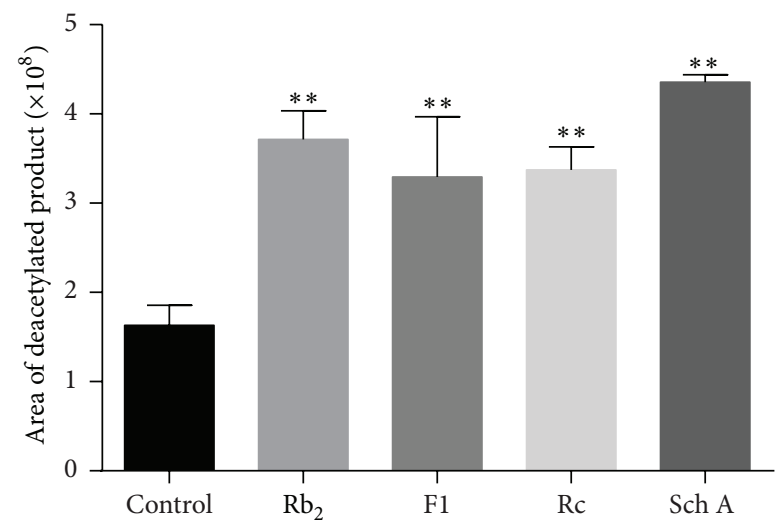

(c)

FIGURE 2: LC-MS results of SIRT1 activation. LC-MS chromatograms in negative ion mode and structures of (a) substrate TPE-GK(Ac)YDD and (b) deacetylated product TPE-GKYDD. (c) Area of deacetylated product in LC-MS chromatogram. SIRT1 was incubated with or without ginsenoside $\mathrm{Rb}_{2}\left(\mathrm{Rb}_{2}\right)$, ginsenoside $\mathrm{F} 1$ ( $\mathrm{F} 1$ ), ginsenoside $\mathrm{Rc}(\mathrm{Rc})$, and schisandrin A ( $\left.\mathrm{Sch} \mathrm{A}\right)$. Each bar represented the mean $\pm \mathrm{SD}$ of triplicate experiments. Compared with control group, ${ }^{* *} p<0.01$. 

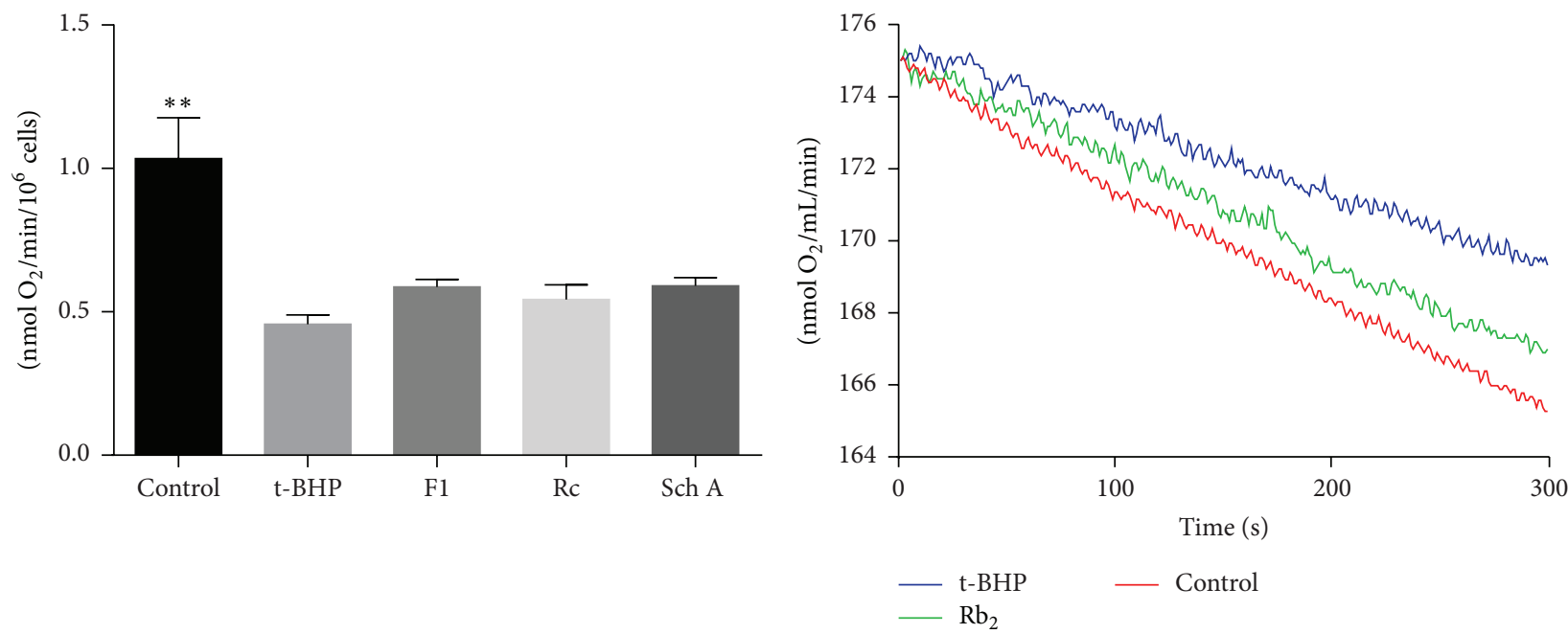

(a)

(b)

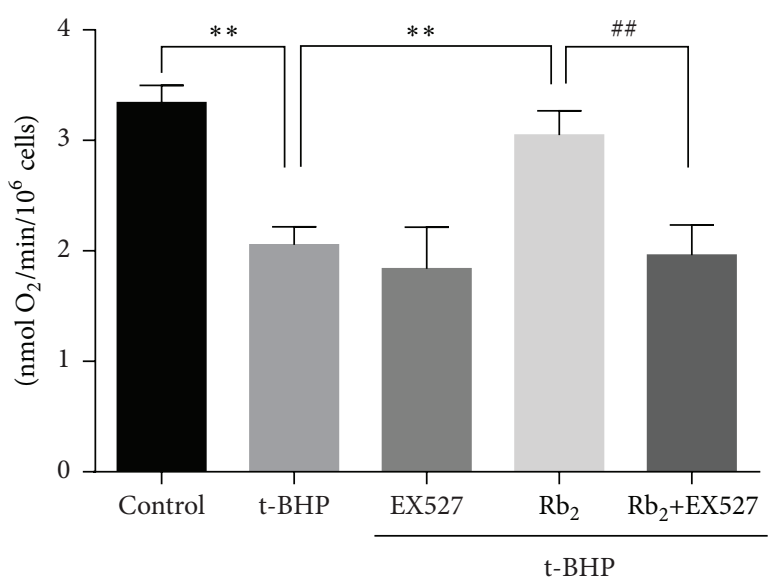

(c)

FIGURE 3: Effects of SIRT1 activators on mitochondrial oxygen consumption. (a) Respiration rates of H9c2 cells, t-BHP injured cells, and tBHP injured cells preincubated with F1, Rc, and Sch A. (b) Representative curves of oxygen consumption recorded by the Clark-type oxygen electrode, and t-BHP injured cells were preincubated with $\mathrm{Rb}_{2}$. (c) In the presence of EX527, the effect of ginsenoside $\mathrm{Rb}_{2}$ was blocked. Each bar represented the mean $\pm \mathrm{SD}$ of triplicate experiments. Compared with $\mathrm{t}-\mathrm{BHP}$ group, ${ }^{*}{ }^{*} p<0.01$. Compared with $\mathrm{t}-\mathrm{BHP}+\mathrm{Rb}_{2}+\mathrm{EX} 527$ group, ${ }^{\# \#} p<0.01$.

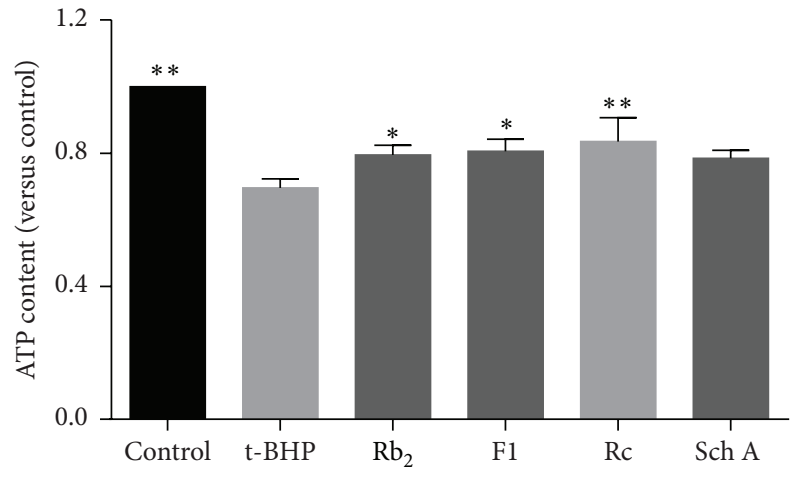

FIGURE 4: Effects of SIRT1 activators on the ATP content in t-BHP treated $\mathrm{H} 9 \mathrm{c} 2$ cells. Each bar represented the mean \pm SD of triplicate experiments. Compared with t-BHP group, ${ }^{*} p<0.05$ and ${ }^{* *} p<$ 0.01 .

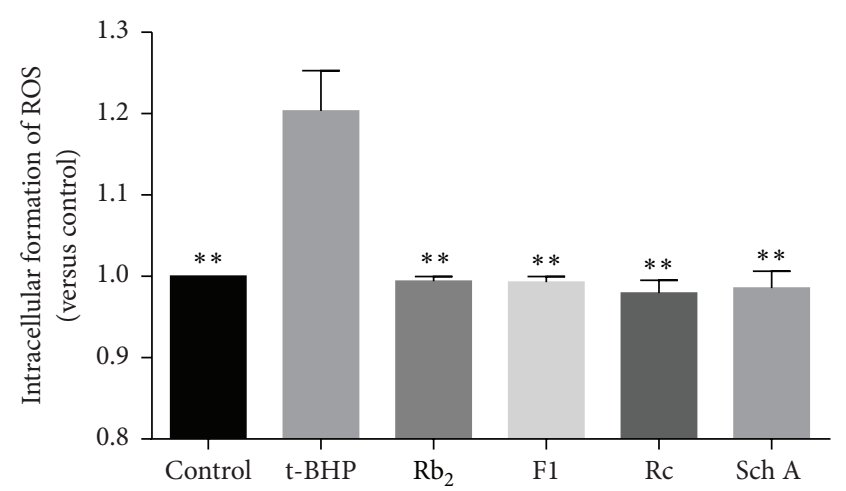

FIGURE 5: Effects of SIRT1 activators on the intracellular ROS level. Each bar represented the mean \pm SD of triplicate experiments. Compared with t-BHP group, ${ }^{* *} p<0.01$. 


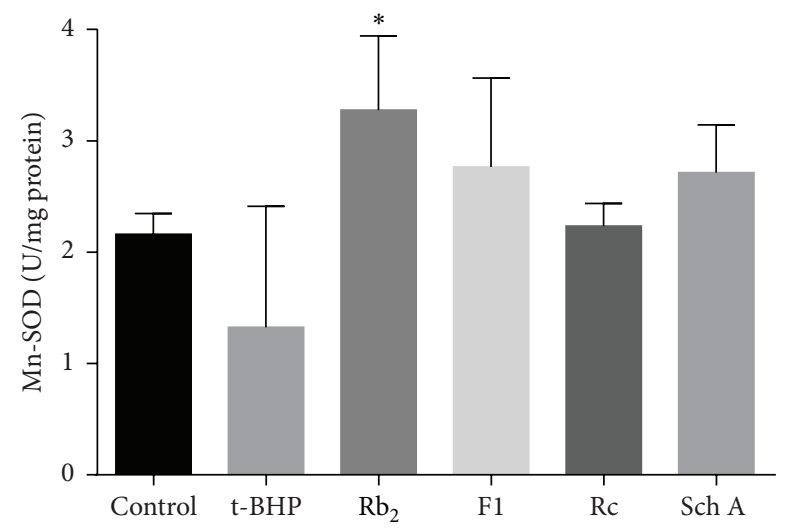

FIGURE 6: Effects of SIRT1 activators on Mn-SOD activity. Each bar represented the mean $\pm \mathrm{SD}$ of triplicate experiments. Compared with t-BHP group, ${ }^{*} p<0.05$.

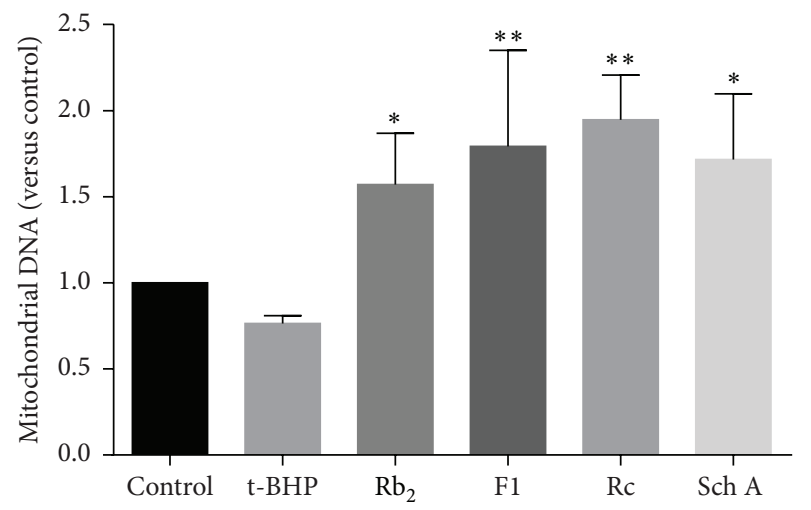

Figure 7: Effects of SIRT1 activators on mitochondrial DNA content. Each bar represented the mean \pm SD of triplicate experiments. Compared with t-BHP group, ${ }^{*} p<0.05$ and ${ }^{* *} p<0.01$.

$\mathrm{Rb}_{3}$, ginsenoside $\mathrm{F} 1$, and ginsenoside Rc from Panax ginseng, ophiopogonin $\mathrm{D}^{\prime}$ from Ophiopogon japonicas, and schisandrin A and schisandrin B from Schisandra chinensis. Interestingly, those herbs consisted of a traditional Chinese formula named Shengmai San, which have been clinically used for the treatment of coronary heart diseases [30,31] and heart failure [32, 33]. Four SIRT1 activators from Shengmai San, including ginsenoside $\mathrm{Rb}_{2}$, ginsenoside $\mathrm{F} 1$, ginsenoside $\mathrm{Rc}$, and schisandrin A, were validated by LC-MS analysis and we found their effects against mitochondrial oxidative damage in further study. Our findings were in accordance with previous reports on other cell lines. Ginsenoside Rc was reported to suppress oxidative stress in HEK293T cells [34], whilst schisandrin A inhibited apoptosis induced by $\mathrm{H}_{2} \mathrm{O}_{2}$ in intestinal epithelial cells [35].

Mitochondrial dysfunction has been one of mechanisms in organ injuries and diseases due to its influence on ATP formation, metabolism, and apoptosis [36]. Our findings indicated that those SIRT1 activators elevated ATP content, prevented ROS formation, and increased the activity of MnSOD. Mitochondrial DNA content and oxygen consumption were also moderated by SIRT1 activators. Those results indicated that SIRT1 activators protected mitochondrial function by improving mitochondrial DNA content, which led to promotion of ATP content, mitochondrial oxygen consumption, and reduction of ROS formation.

\section{Conclusion}

In summary, we identified 19 SIRT1 activators from TCMs. Four active compounds, ginsenoside $\mathrm{Rb}_{2}$, ginsenoside $\mathrm{F}$, ginsenoside Rc, and schisandrin A, exerted significant activities against t-BHP induced oxidative damage in cardiomyocytes. Our findings provided useful evidence to illustrate the cardioprotective effects of TCMs with Tonification effects and led to a new insight into the scientific illustration of TCMs theory.

\author{
Abbreviations \\ TCMs: Traditional Chinese medicines \\ AIE: Aggregation induced emission \\ LC-MS: Liquid chromatography-mass spectrometry \\ t-BHP: tert-Butyl hydroperoxide \\ ROS: Reactive oxygen species \\ F1: $\quad$ Ginsenoside F1 \\ Rc: $\quad$ Ginsenoside Rc \\ Sch A: Schisandrin A \\ $\mathrm{Rb}_{2}$ : Ginsenoside $\mathrm{Rb}_{2}$.
}

\section{Conflict of Interests}

The authors declare that there is no conflict of interests regarding the publication of this paper.

\section{Acknowledgments}

This work was supported by grant from the National Basic Research Program of China (no. 2012CB518405) and the Zhejiang Provincial Natural Science Foundation of China (no. LR16H280001).

\section{References}

[1] H.-C. Chang and L. Guarente, "SIRT1 and other sirtuins in metabolism," Trends in Endocrinology \& Metabolism, vol. 25, no. 3, pp. 138-145, 2014.

[2] L. Zhao, R. An, Y. Yang et al., "Melatonin alleviates brain injury in mice subjected to cecal ligation and puncture via attenuating inflammation, apoptosis, and oxidative stress: the role of SIRT1 signaling," Journal of Pineal Research, vol. 59, no. 2, pp. 230-239, 2015.

[3] S. Masri and P. Sassone-Corsi, "Sirtuins and the circadian clock: bridging chromatin and metabolism," Science Signaling, vol. 7, no. 342, article re6, 2013.

[4] G. Donmez and T. F. Outeiro, "SIRT1 and SIRT2: emerging targets in neurodegeneration," EMBO Molecular Medicine, vol. 5, no. 3, pp. 344-352, 2013.

[5] Z. Ungvari, N. Labinskyy, P. Mukhopadhyay et al., "Resveratrol attenuates mitochondrial oxidative stress in coronary arterial 
endothelial cells," The American Journal of Physiology-Heart and Circulatory Physiology, vol. 297, no. 5, pp. H1876-H1881, 2009.

[6] M. Tan, C. Tang, Y. Zhang et al., "SIRT1/PGC-1 $\alpha$ signaling protects hepatocytes against mitochondrial oxidative stress induced by bile acids," Free Radical Research, vol. 49, no. 8, pp. 935-945, 2015.

[7] Q. Sun, H. Hu, W. Wang, H. Jin, G. Feng, and N. Jia, “Taurine attenuates amyloid $\beta$ 1-42-induced mitochondrial dysfunction by activating of SIRT1 in SK-N-SH cells," Biochemical and Biophysical Research Communications, vol. 447, no. 3, pp. 485489, 2014.

[8] S. Takeo, D. Sato, K. Kimura et al., "Resveratrol improves the mitochondrial function and fertilization outcome of bovine oocytes," The Journal of Reproduction and Development, vol. 60, no. 2, pp. 92-99, 2014.

[9] Y. Yang, W. Duan, Y. Lin et al., "SIRT1 activation by curcumin pretreatment attenuates mitochondrial oxidative damage induced by myocardial ischemia reperfusion injury," Free Radical Biology and Medicine, vol. 65, pp. 667-679, 2013.

[10] V. S. LeBleu, J. T. O'Connell, K. N. Gonzalez Herrera et al., "PGC- $1 \alpha$ mediates mitochondrial biogenesis and oxidative phosphorylation in cancer cells to promote metastasis," Nature Cell Biology, vol. 16, no. 10, pp. 992-1003, 2014.

[11] M. Lagouge, C. Argmann, Z. Gerhart-Hines et al., "Resveratrol improves mitochondrial function and protects against metabolic disease by activating SIRT1 and PGC-1 $\alpha$," Cell, vol. 127, no. 6, pp. 1109-1122, 2006.

[12] K. S. Bhullar and B. P. Hubbard, "Lifespan and healthspan extension by resveratrol," Biochimica et Biophysica Acta (BBA)Molecular Basis of Disease, vol. 1852, no. 6, pp. 1209-1218, 2015.

[13] S. J. Mitchell, A. Martin-Montalvo, E. M. Mercken et al., "The SIRT1 activator SRT1720 extends lifespan and improves health of mice fed a standard diet," Cell Reports, vol. 6, no. 5, pp. 836843, 2014.

[14] K. T. Howitz, K. J. Bitterman, H. Y. Cohen et al., "Small molecule activators of sirtuins extend Saccharomyces cerevisiae lifespan," Nature, vol. 425, no. 6954, pp. 191-196, 2003.

[15] B. P. Hubbard and D. A. Sinclair, "Small molecule SIRT1 activators for the treatment of aging and age-related diseases," Trends in Pharmacological Sciences, vol. 35, no. 3, pp. 146-154, 2014.

[16] R. K. Minor, J. A. Baur, A. P. Gomes et al., "SRT1720 improves survival and healthspan of obese mice," Scientific Reports, vol. 1, article 70, 2011.

[17] B. B. Mishra and V. K. Tiwari, "Natural products: an evolving role in future drug discovery," European Journal of Medicinal Chemistry, vol. 46, no. 10, pp. 4769-4807, 2011.

[18] M. K. Beissenhirtz, R. C. H. Kwan, K. M. Ko, R. Renneberg, F. W. Scheller, and F. Lisdat, "Comparing an in vitro electrochemical measurement of superoxide scavenging activity with an in vivo assessment of antioxidant potential in Chinese tonifying herbs," Phytotherapy Research, vol. 18, no. 2, pp. 149-153, 2004.

[19] R. Chen, H. Shao, S. Lin, J.-J. Zhang, and K.-Q. Xu, "Treatment with Astragalus membranaceus produces antioxidative effects and attenuates intestinal mucosa injury induced by intestinal ischemia-reperfusion in rats," The American Journal of Chinese Medicine, vol. 39, no. 5, pp. 879-887, 2011.

[20] L. Mao, Y. Zhao, A. Niu, and H. Yang, "Effect of Astragalus membranaceus aqueous extract on oxidative stress in exhausting exercise mice," Journal of Food, Agriculture and Environment, vol. 11, no. 2, pp. 18-21, 2013.
[21] T. Ramesh, S.-W. Kim, S.-Y. Hwang, S.-H. Sohn, S.-K. Yoo, and S.-K. Kim, "Panax ginseng reduces oxidative stress and restores antioxidant capacity in aged rats," Nutrition Research, vol. 32, no. 9, pp. 718-726, 2012.

[22] A. H. Farrag, W. E. Abdel-Aal, A. A. El-Kady, and M. A. AbedelWahhab, "Efficacy of Panax ginseng against oxidative stress induced by PCBs in rats," Planta Medica, vol. 74, pp. 1007-1008, 2008.

[23] C. Yan, J. Zhu, X. Jia, C. Wang, S. Wang, and L. Kang, "Panax notoginseng saponin attenuates hypoxia/reoxygenationinduced oxidative stress in cortical neurons," Neural Regeneration Research, vol. 7, no. 36, pp. 2853-2859, 2012.

[24] H. Li, W.-Y. He, F. Lin, and X. Gou, "Panax notoginseng saponins improve erectile function through attenuation of oxidative stress, restoration of akt activity and protection of endothelial and smooth muscle cells in diabetic rats with erectile dysfunction," Urologia Internationalis, vol. 93, no. 1, pp. 9299, 2014.

[25] K.-C. Chen, Y.-R. Jian, M.-F. Sun, T.-T. Chang, C.-C. Lee, and C. Y.-C. Chen, "Investigation of silent information regulator 1 (Sirtl) agonists from Traditional Chinese Medicine," Journal of Biomolecular Structure and Dynamics, vol. 31, no. 11, pp. 12071218, 2013.

[26] Y. Wang, Y. Chen, H. Wang, Y. Cheng, and X. Zhao, "Specific turn-on fluorescent probe with aggregation-induced emission characteristics for SIRT1 modulator screening and living-cell imaging," Analytical Chemistry, vol. 87, no. 10, pp. 5046-5049, 2015.

[27] D. Zhao, Q. Li, Q. Huang et al., "Cardioprotective effect of propofol against oxygen glucose deprivation and reperfusion injury in H9c2 cells," Oxidative Medicine and Cellular Longevity, vol. 2015, Article ID 184938, 8 pages, 2015.

[28] L. Zhu, C. Y. Chiao, K. G. Enzer, A. J. Stankiewicz, D. V. Faller, and Y. Dai, "SIRT1 inactivation evokes antitumor activities in NSCLC through the tumor suppressor p27," Molecular Cancer Research, vol. 13, no. 1, pp. 41-49, 2015.

[29] D. H. Shin, Y.-J. Choi, and J.-W. Park, "SIRT1 and AMPK mediate hypoxia-induced resistance of non-small cell lung cancers to cisplatin and doxorubicin," Cancer Research, vol. 74, no. 1, pp. 298-308, 2014.

[30] Y. Q. Chen and K. J. Chen, "Characteristics of cardiac function and the effect of shengmai injection on qi deficiency and qi-yin deficiency in patients with coronary heart disease," Zhong Xi Yi Jie He Za Zhi, vol. 8, no. 9, pp. 515-526, 1988.

[31] R. Wu, X. Liu, J. Wang, and X. Z. Zhou, "Study on law using Chinese drug of famous old docter of traditional Chinese medicine to coronary heart disease based on association rules," Zhongguo Zhong Yao Za Zhi, vol. 32, no. 117, pp. 1786-1788, 2007.

[32] Q. Zhou, W. Z. Qin, S. B. Liu, J. S. Kwong, J. Zhou, and J. Chen, "Shengmai (a traditional Chinese herbal medicine) for heart failure," Cochrane Database of Systematic Reviews, vol. 4, Article ID CD005052, 2014.

[33] L. Chen, W.-X. Liang, and Z.-P. Lv, "Synthetic evaluation of the clinical effect of the Shengmai capsule for treatment of chronic congestive heart failure using analytic hierarchy process," Nan Fang Yi Ke Da Xue Xue Bao, vol. 30, no. 9, pp. 2036-2040, 2010.

[34] D. H. Kim, C. H. Park, D. Park et al., "Ginsenoside Rc modulates Akt/FoxO1 pathways and suppresses oxidative stress," Archives of Pharmacal Research, vol. 37, no. 6, pp. 813-820, 2014. 
[35] B.-H. Gu, N. Van Minh, S.-H. Lee et al., "Deoxyschisandrin inhibits $\mathrm{H}_{2} \mathrm{O}_{2}$-induced apoptotic cell death in intestinal epithelial cells through nuclear factor- $\kappa \mathrm{B}$," International Journal of Molecular Medicine, vol. 26, no. 3, pp. 401-406, 2010.

[36] J. A. Funk, S. Odejinmi, and R. G. Schnellmann, "SRT1720 induces mitochondrial biogenesis and rescues mitochondrial function after oxidant injury in renal proximal tubule cells," Journal of Pharmacology and Experimental Therapeutics, vol. 333 , no. 2 , pp. 593-601, 2010. 


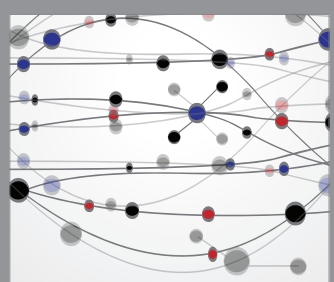

The Scientific World Journal
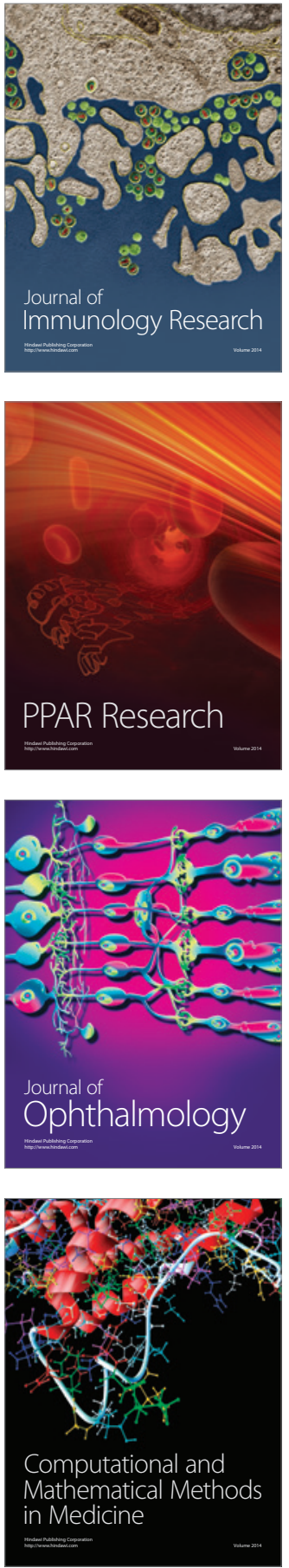

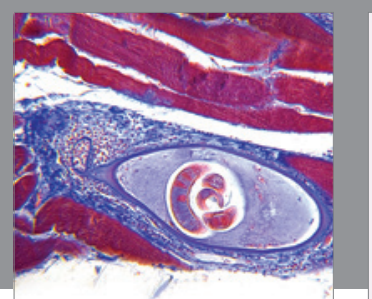

Gastroenterology Research and Practice

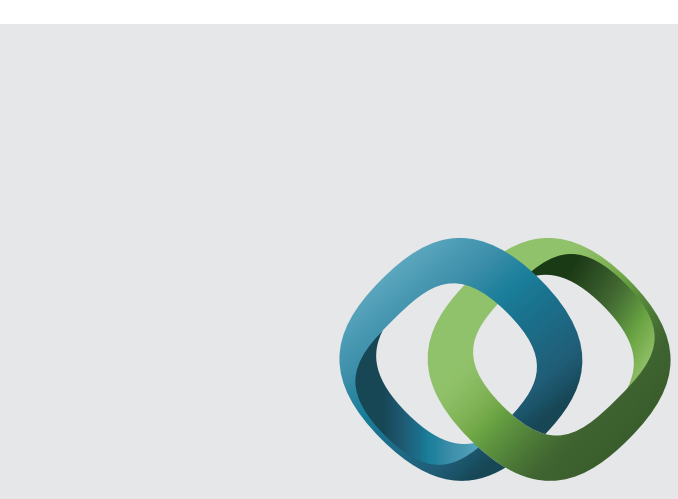

\section{Hindawi}

Submit your manuscripts at

http://www.hindawi.com
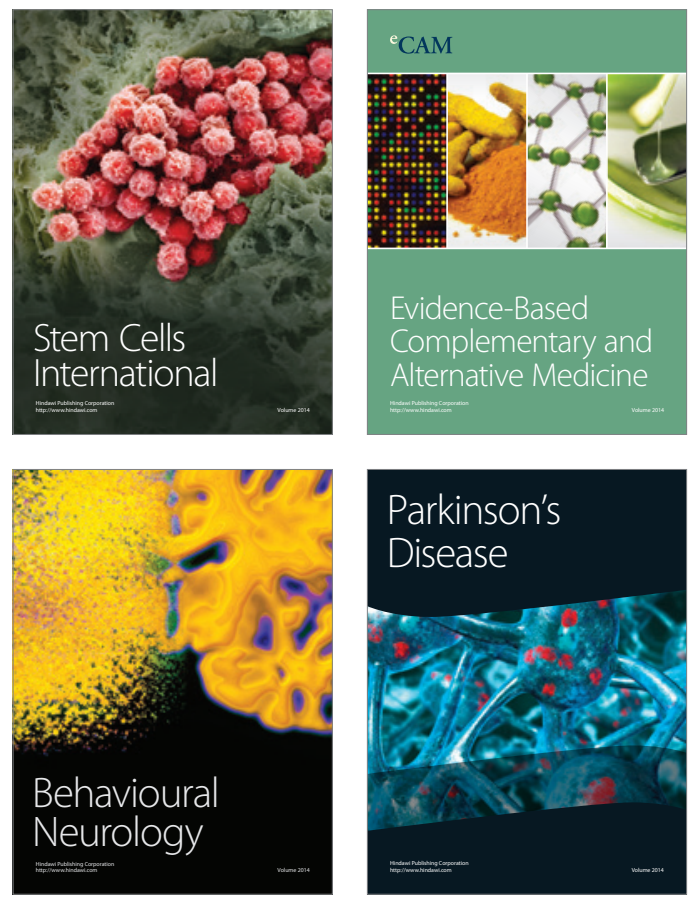
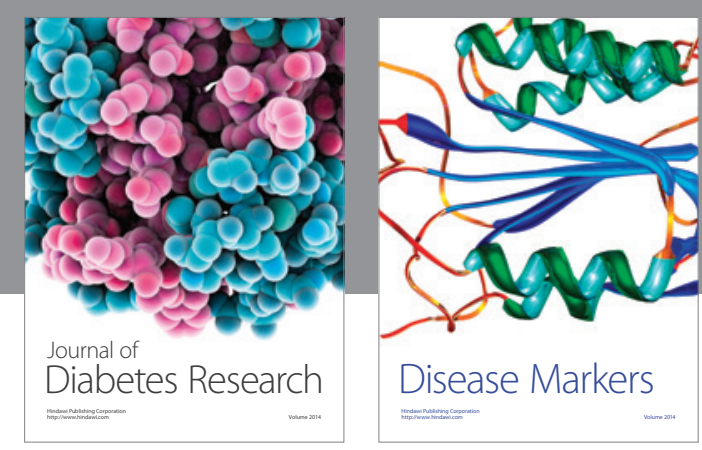

Disease Markers
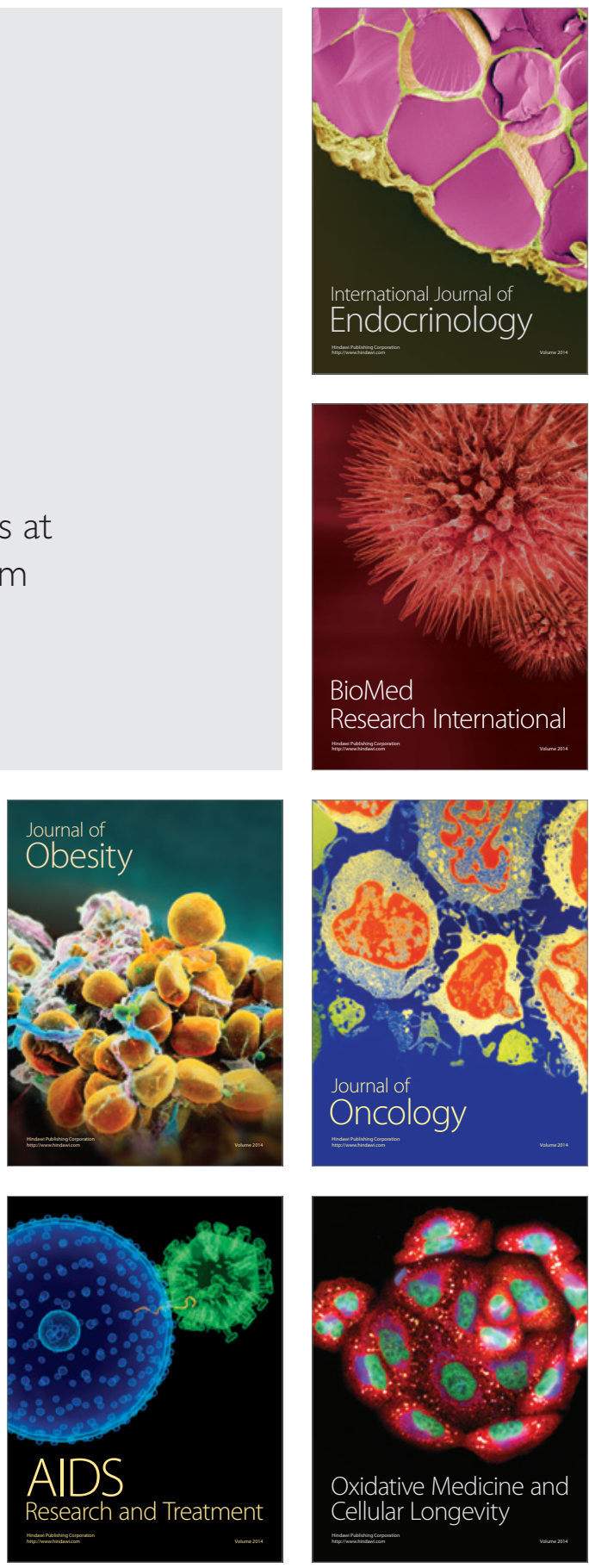